\title{
CORRECTION TO A PAPER OF R. H. BRUCK
}

\author{
BY \\ RICHARD ROTH
}

In his paper in the Transactions Difference sets in a finite group, Bruck gives a proof that no regular transitive plane of order 18 exists [1, p. 470, Example 2]. The proof depends upon showing that certain equations which would have to be solvable by non-negative integers have no such solutions. However the proof is incomplete since there is, in fact, a solution of the equations Bruck considers. This note shows how to dispose of this further case and completes the proof that no transitive regular projective planes of order 18 exist.

For most definitions and theorems involved see Bruck's paper [1]. For further background material on projective planes the reader is referred to [3, Chapter 20].

1. By a regular transitive finite projective plane we mean a plane for which there is a collineation group $G$ which is regular (and transitive) on the points of the plane; i.e., for every pair of points $P$ and $Q$ there is precisely one collineation in $G$ taking $P$ onto $Q$. (Note that Bruck used only the terms "transitive" to mean "regular transitive'”) The corollary to Theorem 4.2, p. 471 in Bruck's article [1] shows that 2 and 3 would be multipliers for a regular transitive plan of order 18 in the case that $G$ is abelian and this can be used to show the nonexistence of the plane; moreover, the discussion which follows for the non-abelian cases is applicable in addition for all possible abelian cases except if $G$ is cyclic and it is well known that no cyclic plane of order 18 exists (see [2]). We thus consider the case that $G$ is non-abelian of order $18^{2}+18+1=343=7^{3}$.

We quote the following proposition which is basic to the discussion $[1$, Lemma 4.1, p. 469].

Let $(G, D)$ be a $(v, k, \lambda)$ difference set. Let $\theta$ be a homomorphism of $G$ upon a group $H$ of order $v_{1}$, and, for each $h \in H$, let $N(h)$ be the number of $d \in D$ such that $d \theta=h$. Then if $v=v_{1} w$,

$$
\begin{aligned}
\sum_{h} N(h) & =k, \\
\sum_{h} N(h)^{2} & =n+\lambda w, \\
\sum_{h} N(h) N\left(h_{1} h\right) & =\lambda w \quad \text { for } h_{1} \neq 1
\end{aligned}
$$

where in each case the sum is over the elements $h$ of $H$.

Received by the editors November 13, 1964. 
In the example in question $v=7^{3}, k=19, \lambda=1, n=18$. Any group of order $7^{3}$ is homomorphic to the additive integers modulo $v_{1}=7$ and equations (1) and (2) become in this case

$$
\begin{aligned}
& \sum N(i)=19, \\
& \sum N(i)^{2}=67
\end{aligned}
$$

where $i$ runs from 0 to 6 . Bruck gives 4 types of solutions $(a),(b),(c),(d)[1$, p. 470] which satisfy these two equations. These 4 cases are then eliminated by means of equations (3) which become

$$
\sum_{i} N(i) N(i+j)=49, \quad j=1,2,3,
$$

( $i$ runs from 0 to 6 ). This is facilitated by using congruences modulo 3 .

However there are two more types of solutions to equations (1) and (2), namely

$$
\begin{aligned}
& n_{0}=1, n_{1}=1, n_{3}=2, n_{4}=3, \\
& n_{1}=3, n_{4}=4 .
\end{aligned}
$$

Here $n_{j}$ is the number of $N(i)$ having value $j$ (i.e. $n_{2}=3$ means that three 2's appear in the collection of numbers $N(i)$ ).

Type (e) may be eliminated by equations (6) in a similar fashion to the 4 cases (a) through (d). However $N(0)=N(1)=N(3)=1, N(2)=N(4)=N(5)=N(6)=4$ of type (f) will be seen to satisfy equations (6) also. Further work is needed to eliminate type (f).

2. There are two non-abelian groups of order $7^{3}=343$, both have centers $Z$ of order 7 and $G / Z$ in both cases is elementary abelian of order 49 (see [3, pp. 51-52]). (Also any noncyclic abelian group of order $7^{3}$ has a subgroup $Z$ with the same property.) Thus $G$ is also homomorphic to a group of order 49 and equations (1), (2) become

$$
\begin{aligned}
& \sum_{h} N(h)=19, \\
& \sum_{h} N(h)^{2}=25
\end{aligned}
$$

where $h$ runs over the 49 elements of $G / Z$. Possible solutions to these two equations are of the types

$$
\begin{aligned}
& \left(n_{0}=32\right), n_{1}=16, n_{3}=1, \\
& \left(n_{0}=33\right), n_{1}=13, n_{2}=3,
\end{aligned}
$$

$G / Z$ is the direct product of 2 cyclic groups of order 7 . We may represent the elements of $G / Z$ in the form $a^{i} b^{j}, i, j=0, \cdots 6$, where $a$ and $b$ are generators of the two groups. Each element of $G / Z$ is a coset of $G$ modulo $Z$ and we may 
form a $7 \times 7$ array of numbers $N_{i j}$ such that $N_{i j}$ is the number of elements in the difference set which belong to the coset $a^{i} b^{j}$. These are precisely the $N(h)$ 's corresponding to the homomorphism $G \rightarrow G / Z$.

Thus in case $(\alpha)$ this array will be filled with sixteen 1's and one 3 ; in case $(\beta)$ there will be thirteen 1's and three 2's. All the rest will be 0's.

Any subgroup $\bar{H}$ of $G / Z$ of order 7 corresponds to a normal subgroup $H$ of $G$ of order 49 and the mapping $G \rightarrow G / H$ must give rise to the equations (4), (5), (6) when we consider how the elements of the difference set are mapped. Each of the elements $a, b, a b, a b^{2}, a b^{3}, a b^{4}, a b^{5}, a b^{6}$ generates a different subgroup $\bar{H}$ and each element $a^{i} b^{j}$ not the identity belongs to one such subgroup. Thus the positions in our $7 \times 7$ array other than the one corresponding to the identity are partitioned into 8 disjoint sets $S_{i}$ of 6 positions each. Any $N_{00}$ plus the $N_{i j}$ 's in the 6 other positions corresponding to an $\bar{H}$ add up to the number of difference set elements mapped onto the identity under the homomorphism $G \rightarrow G / H$, the latter being a group of order 7 . We have seen this must be either 1 or 4 ("case $f$ " in previous discussion).

Consider case ( $\alpha$ ). We may assume that $N_{00}=3$. (If not, an equivalent difference set is obtained by multiplying all of $G$ by some element of $G$ which "translates" the 3 to the $N_{00}$ position.) There are sixteen 1's that remain to be distributed among the 48 positions. But each $S_{i}$ must only receive exactly one 1 (to add up to 4 ) thus only eight 1 's may be assigned. Hence case $(\alpha)$ is ruled out.

In case ( $\beta$ ) we may assume that $N_{00}=2$. There remain two 2's and thirteen 1's to distribute. Since the numbers in each set $S_{i}$ together with $N_{00}$ adds up to 4 we may assign the two 2's to two of the $S_{i}$ 's and two 1's to each of the 6 other $S_{i}$ 's. This uses only twelve 1 's and there is no place for the remaining 1 . Thus case $(\beta)$ is eliminated and this completes the proof.

\section{BIBLIOGRAPHY}

1. R. H. Bruck, Difference sets in a finite group, Trans. Amer. Math. Soc. 78 (1955), 464-481.

2. Marshall Hall, Jr., Cyclic projective planes, Duke Math. J. 14 (1947), 1079-1090.

3. —, The theory of groups, Macmillan, New York, 1959.

UNIVERSITY OF COLORADO,

BOULDER, COLORADO 